\title{
Incorporación de la promoción y educación para la salud en los planes de estudio de grado en Ciencias de la Salud de la Universitat de València
}

\author{
Luisa Ruano-Casado, M. Luisa Ballestar-Tarín
}

Introducción. En el presente trabajo se analiza la importancia de la formación para el desarrollo de la promoción y educación para la salud, siendo imprescindible disponer de profesionales con una capacitación eficiente y de calidad, coherente con sus funciones y las necesidades en salud de la población. El objetivo es identificar el grado de incorporación a los planes de estudio de grado, la formación del estudiantado en promoción y educación para la salud en las titulaciones de las ramas de salud de la Universitat de València.

Sujetos y métodos. Se realiza un análisis de contenido en los planes de estudio y de las asignaturas de las once titulaciones, a través de las guías académicas dispuestas en la red de la Universitat de València en el curso 2012-2013.

Resultados. La formación en promoción y educación para la salud apenas se contempla en las competencias generales y específicas de los planes de estudios, y en ninguno de ellos se muestran reflejadas las competencias básicas y transversales. De las 519 asignaturas, la promoción y educación para la salud sólo aparece en 54 asignaturas, siendo el grado de Enfermería la titulación que más formación imparte a sus estudiantes.

Conclusión. A pesar de las recomendaciones de las diversas entidades internacionales y nacionales de la importancia en formación en promoción y educación para la salud para reducir las desigualdades en salud, su visibilidad es muy escasa.

Palabras clave. Ciencias de la salud. Guías académicas, Formación. Planes de estudio. Promoción y educación para la salud.

Promotion and health education incorporation in the undergraduate study plan in Health Science of the University of Valencia

Introduction. The present article discusses the significance of teaching for the development of the promotion and health education. It is important to have professionals with an efficient and good quality formation, consistent with their roles and the population health necessities. The objective sought is to recognize the incorporation into undergraduate curricula as well as training of students in promotion and health education degrees into the health fields of the University of Valencia.

Subjects and methods. Curriculum and subjects analysis of eleven degrees as well as academic guides examination arranged in the network of the University of Valencia in the year 2012-2013.

Results. It has been observed reduced study plan in general and specific competencies. In terms of the degree, there is no concept reflected in the basic and transversal capabilities. Among the 519 subjects, promotion and health education appears in 54 subjects only, being the Nursing degree the one that provides more training to the students.

Conclusion. Although several international and national organizations recommend the importance of training in promotion and health education to reduce health discrepancies, its visibility is minimal.

Key words. Academic guide. Curriculum. Health sciences. Promotion and health education. Training.

\section{Introducción}

La formación es un elemento esencial para el ejercicio correcto y eficaz de la promoción de salud y la práctica de la educación para la salud. Esta formación debe situarse al mismo nivel que la del tratamiento de las enfermedades. Es imprescindible disponer de una formación suficiente y de calidad, co- herente con las funciones y el papel de los profesionales de la salud [1], para resolver las desigualdades en salud.

En las últimas décadas, la necesidad de la formación en promoción y educación para la salud viene recogida por distintas instituciones y en diversos documentos, tanto de ámbito nacional como internacional. La Organización Mundial de la Salud (OMS)
Departamento de Enfermería. Facultad de Enfermería y Podología. Universitat de València. Valencia, España.

Correspondencia: Prof. Luisa Ruano Casado. Departamento de Enfermería. Facultad de Enfermería y Podología. Universitat de València. Jaime Roig, s/n. E-46010 Valencia.

E-mail: luisa.ruano@uv.es Conflicto de intereses: No declarado.

Competing interests: None declared.

(c) 2015 FEM 
[2] formuló en 1977, en su estrategia 'Salud para todos en el año 2000', un objetivo dirigido a desarrollar recursos humanos en salud. En esta misma línea, el informe 'SESPAS 2000' señala que la educación-formación del personal sanitario debería contribuir activamente a alcanzar los objetivos de salud para todos. Sin embargo, la evaluación posterior indica que, en ocasiones, se produce un desajuste entre formación recibida y requerimientos del trabajo [3].

En 1978, la OMS [4], reunida en Alma-Ata, adoptó la atención primaria de salud (APS) como estrategia válida para el logro de la salud para todos. En nuestro país, el trabajo de los equipos de APS se debe fundamentar y organizar en el desarrollo de actividades de promoción de la salud y de actuaciones preventivas.

Estos mismos principios se defienden en la Ley General de Sanidad 14/1986 de 25 de abril [5], la Ley 44/2003, de Ordenación de las Profesiones Sanitarias [6], y la Ley Orgánica 16/2003, de Cohesión y Calidad del Sistema Nacional de Salud [7].

En la 'Carta de Ottawa', como resultado de la I Primera Conferencia Internacional de Promoción de la Salud (1986), se identificaron los campos de acción: políticas públicas saludables, ambientes favorables a la salud, acción comunitaria, habilidades personales y reorientación del sistema de salud. Se recomienda la participación comunitaria en los procesos decisorios de la planificación y de la implementación de las acciones de salud [8], donde la creación de entornos que apoyen la salud es prioritaria para promocionar la salud. Este aspecto es fundamental para entender el movimiento de universidades promotoras de salud.

En el año 2000 tuvo lugar la conferencia de México [9], donde se manifiesta que para asegurar la infraestructura necesaria para la promoción de la salud es necesario 'el desarrollo de recursos humanos por medio de la educación, el adiestramiento y el intercambio de experiencias. Las universidades y otras instituciones educacionales tienen una función de vital importancia al asegurar que una amplia gama de profesiones tengan contacto con los conceptos y estrategias de promoción de la salud'.

Asimismo, en el objetivo 18 del informe 'Salud 21', dirigido a desarrollar los recursos humanos para la salud, declara que 'para el año 2010, todos los estados miembros deberán garantizar que los profesionales sanitarios y de otros sectores han adquirido los conocimientos, las actitudes y las cualificaciones necesarios para proteger y promover la salud' [10].

Para hacer frente a estos desafíos, en la formación de los estudios de grado impartidos por las universidades se hace imprescindible más aún, si ésta adquiere el compromiso de potenciar los principios, valores y propuestas de promoción de la salud que se recogen en la 'Carta de Edmonton' para universidades promotoras de la salud e instituciones de educación superior [11].

El movimiento de universidades promotoras de la salud aparece a finales de los años noventa, en el I Congreso Internacional de Universidades Promotoras de la Salud celebrado en Santiago de Chile. El tema central del evento fue 'Construyendo universidades saludables'.

En España se celebró el IV Congreso Internacional de Universidades Promotoras de la Salud en 2009, en la Universidad Pública de Navarra, en el que se aprobó un documento resolutivo conocido como 'Declaración de Pamplona/Iruña'. El documento establece los valores y principios del movimiento de universidades promotoras de la salud y plantea como estrategia la incorporación de la formación en promoción de la salud en los planes de estudio universitario de grado y posgrado [12].

En la Asamblea General de la Red Iberoamericana de Universidades Promotoras de la Salud (RIUPS) se aprobó el 'desarrollo de acciones de formación académica profesional en promoción de la salud, determinantes sociales de la salud, educación para la salud y otras modalidades de capacitación a la comunidad universitaria' [13].

La Red Española de Universidades Saludables se constituyó el 22 de septiembre de 2008. El Ministerio de Sanidad, Servicios Sociales e Igualdad, el Ministerio de Educación, Cultura y Deporte y la Conferencia de Rectores de las Universidades Españolas apoyan y favorecen el desarrollo de esta red, potencian el desarrollo de los proyectos de universidades saludables e invitan a unirse a esta red a todas las universidades interesadas [14]. En esta red participa la Universitat de València, dando respuesta a la quinta área de acción de la carta de Ottawa de promoción de la salud.

Sin embargo, se desconoce la formación que están recibiendo los profesionales de la salud en este ámbito [15], la influencia que tiene la universidad en los futuros profesionales de la salud pública [16] y si esta formación contribuye a mejorar la efectividad, eficiencia y equidad de los recursos, del empoderamiento por parte de las personas y colectivos, para el control de los determinantes de la salud [15].

Si se atiende a estos criterios, el objetivo es identificar hasta qué punto se ha incorporado, a los planes de estudio de grado, la formación del alumnado en promoción y educación para la salud en las titulaciones de las ramas de salud en la Universitat de València. 


\section{Sujetos y métodos}

La metodología empleada es el análisis de contenido. Se ha analizado la información de los planes de estudio y asignaturas de la rama de ciencias de la salud en las titulaciones de grado en Ciencia y Tecnología de los Alimentos, Enfermería, Farmacia, Fisioterapia, Logopedia, Medicina, Nutrición Humana y Dietética, Odontología, Óptica y Optometría, Podología y Psicología [17] del curso 2012-2013.

Para ello se han utilizado los descriptores de 'promoción', promover,' 'educación' y 'educar', en las competencias básicas, generales, transversales y específicas de los planes de estudio de las 11 titulaciones y en las asignaturas que contemplan la promoción y educación para la salud, en los distintos apartados de las guías didácticas: resumen, competencias, resultados de aprendizaje y contenidos de las mismas.

\section{Resultados}

Las 11 titulaciones analizadas recogen 519 asignaturas (excluyendo la asignatura de 'Trabajo fin de grado') que entre todas ellas suman 2.880 créditos. La promoción y educación para la salud se contempla en ocho titulaciones y está considerada entre las competencias generales en las titulaciones de Enfermería, Farmacia, Fisioterapia, Medicina, Odontología y Podología; salvo en Enfermería, sólo se incluye en una o dos competencias generales y en competencias específicas en Farmacia y Fisioterapia. En ninguna titulación aparece en las competencias básicas y transversales (Tabla I).

La promoción y educación para la salud figura en las distintas titulaciones como una competencia general que debe alcanzar el alumnado a partir del desarrollo de propuestas y estrategias de intervención, como por ejemplo: 'Plantear y proponer las medidas preventivas adecuadas a cada situación clínica: cuidar de los problemas de salud de los pacientes, la familia y la comunidad de una manera efectiva, eficiente y de acuerdo con los principios deontológicos, con especial énfasis en la promoción de la salud y la prevención de la enfermedad, formando equipos multidisciplinarios' (competencia general 21, Grado de Medicina).

El análisis de las guías académicas de las asignaturas muestra un panorama diferenciado para cada grado (Tabla II), destacando que tan sólo 54 asignaturas contemplan la promoción y educación para la salud, y únicamente se refleja el contenido en 47 temas de 22 asignaturas. Las titulaciones de Enfer-
Tabla I. Análisis de contenido de las competencias que contemplan la promoción y educación para la salud en los planes de estudio de las diferentes titulaciones.

\begin{tabular}{|c|c|c|c|c|}
\hline & \multicolumn{4}{|c|}{ Competencias } \\
\hline & Básicas & Generales & Transversales & Específicas \\
\hline Ciencia y Tecnología de los Alimentos & - & - & - & - \\
\hline Enfermería & - & $\begin{array}{c}\text { CG13, CG14, CG24, } \\
\text { CG26, CG32, G34 }\end{array}$ & - & CE4 \\
\hline Farmacia & - & CG16 & - & $\begin{array}{c}\text { CE134, CE144, CE150 } \\
\text { CE151, CE325 }\end{array}$ \\
\hline Fisioterapia & - & CG3, CG13 & - & $\begin{array}{l}\text { CE21, CE76, CE80, } \\
\text { CE87, CE88 }\end{array}$ \\
\hline Logopedia & - & - & - & - \\
\hline Medicina & - & CG21 & - & CE102 \\
\hline Nutrición Humana y Dietética & - & - & - & CE14 \\
\hline Odontología & - & CG29 & - & - \\
\hline Óptica y Optometría & - & - & - & CE59, CE118, CE149 \\
\hline Podología & - & CG1, CG6 & - & - \\
\hline Psicología & - & - & - & - \\
\hline
\end{tabular}

mería y de Nutrición y Dietética son las que ofrecen más formación al estudiantado; en sentido contrario, el alumnado que recibe menos o ninguna formación es el de Psicología y Logopedia.

\section{Discusión}

A partir de los resultados obtenidos se podría concluir que es escasa la formación en promoción y educación para la salud en todas las titulaciones de la rama de ciencias de la salud, contemplándose esta formación sólo en 54 asignaturas. Del mismo modo, en dichas asignaturas existen desajustes entre los apartados de las guías académicas, es decir, entre el resumen, las competencias, los resultados de aprendizaje y el contenido.

En comparación con otros estudios de nuestro país, la integración en los currículos en la última década ha sido pobre. Según el informe SESPAS [18], analizados 131 planes de estudio en la educación universitaria, la promoción de la salud y educación para la salud tiene una importancia curricular bastante deficiente. La educación para la salud 
Tabla II. Análisis de contenido de las guías académicas de las asignaturas que contemplan la promoción y educación para la salud, en las diferentes titulaciones.

\begin{tabular}{|c|c|c|c|c|c|}
\hline & Asignaturas $^{\mathrm{a}}$ & Resumen ${ }^{b}$ & Competencias ${ }^{c}$ & $\begin{array}{c}\text { Resultados } \\
\text { de aprendizaje }\end{array}$ & Contenidos $\mathrm{e}$ \\
\hline Ciencia y Tecnología de los Alimentos & 1 & 1 & 1 & - & $1(2)$ \\
\hline Enfermería & 11 & 7 & 4 & 4 & $4(16)$ \\
\hline Farmacia & 5 & 2 & - & 3 & $4(7)$ \\
\hline Fisioterapia & 9 & 6 & 1 & 7 & $1(7)$ \\
\hline Logopedia & 4 & 1 & 2 & - & - \\
\hline Medicina & 3 & - & 1 & - & $3(3)$ \\
\hline Nutrición Humana y Dietética & 5 & 1 & 4 & - & $4(9)$ \\
\hline Odontología & 4 & - & 4 & 2 & $2(3)$ \\
\hline Óptica y Optometría & 4 & - & 3 & - & $1(3)$ \\
\hline Podología & 4 & 3 & 2 & 1 & $1(2)$ \\
\hline Psicología & 4 & 2 & 3 & - & $1(1)$ \\
\hline Totales & $54 / 519$ & 23 & 25 & 17 & $22(47)$ \\
\hline
\end{tabular}

a Número de asignaturas en las que aparecen los descriptores; ${ }^{b}$ Número de asignaturas en las que los descriptores aparecen en el apartado resumen; ${ }^{c}$ Número de asignaturas en las que los descriptores aparecen en el apartado de competencias; ${ }^{d}$ Número de asignaturas en las que los descriptores aparecen en el apartado de resultados de aprendizaje; ' Número de asignaturas en las que los descriptores aparecen en el apartado de contenidos (número de temas).

sólo aparecía en las carreras de medicina, fisioterapia, terapia ocupacional y enfermería.

En América Latina, la información obtenida es de formación de programas de licenciados en promoción y educación para la salud en las universidades de Costa Rica, Universidad Nacional Autónoma de México, Universidad de Nariño (Colombia), Universidad de El Salvador y Universidad de Chimborazo (Ecuador) [19].

Sería necesario investigar qué ocurre en otras titulaciones, además de constatar los resultados con entrevistas a los coordinadores de las asignaturas.

Por tanto, a la vista de los resultados, se observa falta de compromiso por parte de los responsables de los planes de estudio de las diferentes titulaciones en la promoción y educación para la salud, instaurados los planes de estudio en un modelo de enfermedad/curación, sin tener en cuenta las recomendaciones de los diferentes estamentos educativos y en salud.

Es imprescindible capacitar al alumnado y al profesorado desde una visión activa de promoción de la salud, incluyéndose este modelo en los planes de estudio de grado, fomentando competencias transversales en los diferentes planes de formación, o bien incluyendo las asignaturas de promoción y educación para la salud como troncal u optativa.

Se requiere un compromiso por parte de la Conferencia de Rectores de la Universidades Españolas y las redes comunitarias para fomentar proyectos de trabajo que incorporen la identificación de las necesidades de la comunidad universitaria, el concepto de promoción de la salud en la vida universitaria, en sus políticas institucionales, y la incorporación en los procesos y planes de estudio.

Además, es necesario implicar a los sectores de la educación y la sanidad para establecer los principales desafíos y medidas que aborden los determinantes de la salud en un mundo globalizado, desarrollando redes de colaboración global, fomentando una cultura crítica transparente y estableciendo sinergias que multipliquen los resultados de los esfuerzos de todos los agentes implicados, en el que resulta crucial el conocimiento científico [15] en la 
formación de los profesionales para contribuir a la mejora de la salud de la población. La universidad ha venido formando a profesionales de la salud con gran efectividad, pero no es suficiente.

\section{Bibliografía}

1. Ministerio de Sanidad y Consumo. Formación en promoción y educación para la salud. Informe del Grupo de Trabajo de Promoción de la Salud a la Comisión de Salud Pública del Consejo Interterritorial del Sistema Nacional de Salud. Madrid: Ministerio de Sanidad y Consumo; 2003.

2. Organización Mundial de la Salud. Estrategia mundial de salud para todos en el año 2000. Geneva: OMS; 1981.

3. González López-Valcárcel B. Objetivo 36: desarrollar recursos humanos en salud. In: Sociedad Española de Salud Pública y Administraciones Sanitarias. Informe SESPAS 2000: la salud pública ante los desafíos de un nuevo siglo. Granada: Escuela Andaluza de Salud Pública; 1999.

4. Organización Mundial de la Salud. Atención primaria en salud. Informe de la Conferencia Internacional sobre Atención Primaria en Salud. Geneva: OMS; 1978.

5. Ley $14 / 1986$, de 25 de abril, General de Sanidad. Boletín Oficial del Estado, n. ${ }^{\circ}$ 102, de 29 de abril de 1986.

6. Ley 44/2003, de 21 de noviembre, de Ordenación de las Profesiones Sanitarias. Boletín Oficial del Estado, n. ${ }^{\circ} 280$, de 22 de noviembre de 2003.

7. Ley $16 / 2003$, de 28 de mayo, de Cohesión y Calidad del Sistema Nacional de Salud. Boletín Oficial del Estado, n. ${ }^{\circ} 128$, de 29 de mayo de 2003.

8. World Health Organization. Ottawa Charter for Health Promotion. Ottawa: Canadian Public Health Association; 1986.

9. Organización Mundial de la Salud. Quinta Conferencia Mundial de Promoción de la Salud. Promoción de la salud: hacia una mayor equidad. Ciudad de México: OMS; 2000. URL: http://www.who.int/healthpromotion/conferences/ previous/mexico/en/hpr_mexico_report_sp.pdf.

10. Organización Mundial de la Salud. Salud 21: el marco político de salud para todos de la región europea de la OMS (1999). Madrid: Ministerio de Sanidad y Consumo/OMS.

11. Carta de Edmonton para Universidades Promotoras de la Salud e Instituciones de Educación Superior. In: Guía para universidades saludables y otras instituciones de educación superior. 2006. URL: http://www.uc.cl/enfermeria/html/ noticias/doc/guia\%20USal.pdf.

12. Declaración de Pamplona/Iruña. IV Congreso Internaciona de Universidades Promotoras de Salud. 2009. URL: https:// www.myctb.org/wst/consorciointeramericano/Lists/ Anuncios/ Attachments/6/Declaraci\%C3\%B3n\%20de\%20 Pamplona\%20IV\%20Congreso.pdf.

13. Declaración conmemorativa de los diez años del Movimiento de Universidades Promotoras de la Salud en la Región de las Américas (2003-2013). III Asamblea General de la Red Iberoamericana de Universidades Promotoras de la Salud (RIUPS). 2013. URL: http://bit.ly/12au8w1.

14. Red Española de Universidades Saludables (REUS), principios, objetivos y estructuras de la red. Madrid: Ministerio de Sanidad, Servicios Sociales e Igualdad; 2008. URL: http://www.msssi. gob.es/profesionales/saludPublica/prevPromocion/promocion/ UniversidadesSaludables/REUS.htm.

15. Segura-Benedicto A. Una reforma global de la formación de los profesionales. Educ Med 2011; 14: 15-7.

16. Obradors-Ría N, Segura-Benedicto A. La salud pública en las facultades de medicina y escuelas de enfermería de Cataluña. La visión del alumnado. Educ Med 2010; 13: 113-22.

17. Universitat de València. Oferta de grados. URL: http://www. uv.es/uvweb/universitat/ca/estudis-grau/oferta-graus/ofertagraus-1285846094474.html. [01.03.2013].

18. Gómez L, Gascón L, Gallego J, Febrel M, Granizo C. Objetivo 15: mejorar conocimientos y motivaciones para una conducta saludable. In: Sociedad Española de Salud Pública y Administraciones Sanitarias. Informe SESPAS 2000: la salud pública ante los desafíos de un nuevo siglo. Granada: Escuela Andaluza de Salud Pública; 1999.

19. Arroyo HV. La formación de recursos humanos y el desarrollo de competencias para la capacitación en promoción de la salud en América Latina. IUHP Global Health Promotion 2009; 16: 66-72. 